Bull. Korean Math. Soc. 49 (2012), No. 5, pp. 949-959

http://dx.doi.org/10.4134/BKMS.2012.49.5.949

\title{
CLASS-PRESERVING AUTOMORPHISMS OF GENERALIZED FREE PRODUCTS AMALGAMATING A CYCLIC NORMAL SUBGROUP
}

\author{
Wei Zhou And Goansu Kim
}

\begin{abstract}
In general, a class-preserving automorphism of generalized free products of nilpotent groups, amalgamating a cyclic normal subgroup of order 8 , need not be an inner automorphism. We prove that every classpreserving automorphism of generalized free products of finitely generated nilpotent groups, amalgamating a cyclic normal subgroup of order less than 8 , is inner.
\end{abstract}

\section{Introduction}

An automorphism $\alpha$ of a group $G$ is called a class-preserving (or conjugating) automorphism if, for each $g \in G, \alpha(g)$ and $g$ are conjugate in $G$. Burnside [3] constructed a group of order $3^{6}$ admitting class-preserving automorphisms which are not inner. Also Wall [11] constructed a group of order 32 having the same property. On the other hand, Grossman [6] defined that a group $G$ has Property $A$ if all class-preserving automorphisms of $G$ are inner. She proved that free groups and fundamental groups of compact orientable surfaces have Property A. Segal [10] constructed a finitely generated torsion-free nilpotent group which does not have Property A. However, Endimioni [5] showed that free nilpotent groups have Property A.

In [1], it was shown that generalized free products of two free groups, amalgamating a maximal cyclic subgroup, have Property A. Recently, this result was improved, in [13], that tree products of finitely generated nilpotent or free groups, amalgamating infinite cyclic subgroups, have Property A. However, there exists a generalized free product of nilpotent groups, amalgamating a cyclic normal subgroup of order 8, which has not Property A (Example 5.4).

Received May 11, 2011.

2010 Mathematics Subject Classification. Primary 20E26, 20E06, 20 F34.

Key words and phrases. class-preserving automorphisms, generalized free products, nilpotent groups, residually finite.

The first author was supported by Natural Science Foundation Project of CQ(CSTC, 2009BB8111).

This work was supported by the Korea Research Foundation Grant (KRF-2009-013C00002). 
In this paper we show that generalized free products of finitely generated nilpotent groups, amalgamating a cyclic normal subgroup of order less than 8 , have Property A.

Since Grossman [6] proved that outer automorphism groups of finitely generated conjugacy separable groups with Property A are residually finite, the groups mentioned above with Property A have residually finite outer automorphism groups.

\section{Preliminaries}

Throughout this paper we use standard notation and terminology.

If $A$ and $B$ are groups, then $A *_{H} B$ denotes the generalized free product of $A$ and $B$ amalgamating $H . x \sim_{G} y$ means that $x$ and $y$ are conjugate in $G$, otherwise $x \chi_{G} y$. Inn $g$ denotes the inner automorphism of $G$ induced by $g \in G$. Out $(G)$ denotes the outer automorphism group, $\operatorname{Aut}(G) / \operatorname{Inn}(G)$, of $G$. $C_{G}(g)$ denotes the centralizer of $g$ in $G$ and $Z(G)$ denotes the center of $G$.

Definition 2.1. By a class-preserving (or conjugating) automorphism of a group $G$ we mean an automorphism $\alpha$ which is such that, for each $g \in G$, there exists $k_{g} \in G$, depending on $g$, so that $\alpha(g)=k_{g}^{-1} g k_{g}$.

Definition 2.2 ([6]). A group $G$ has Property $A$ if for each class-preserving automorphism $\alpha$ of $G$, there exists a single element $k \in G$ such that $\alpha(g)=$ $k^{-1} g k$ for all $g \in G$, i.e., $\alpha=\operatorname{Inn} k$.

We give some known results which are of fundamental importance for our purpose. Amongst these the following theorem plays an important part in the study of conjugate class in generalized free products.

Theorem 2.3 ([8, Theorem 4.6]). Let $G=A *_{H} B$ and let $x \in G$ be of minimal length in its conjugacy class. Suppose that $y \in G$ is cyclically reduced, and that $x \sim_{G} y$.

(1) If $\|x\|=0$, then $\|y\| \leq 1$ and, if $y \in A$, then there is a sequence $h_{1}, h_{2}, \ldots, h_{r}$ of elements in $H$ such that $y \sim_{A} h_{1} \sim_{B} h_{2} \sim_{A} \cdots \sim h_{r}=x$.

(2) If $\|x\|=1$, then $\|y\|=1$ and, either $x, y \in A$ and $x \sim_{A} y$, or $x, y \in B$ and $x \sim_{B} y$.

(3) If $\|x\| \geq 2$, then $\|x\|=\|y\|$ and $y \sim_{H} x^{*}$, where $x^{*}$ is a cyclic permutation of $x$.

Theorem 2.4 ([6, Grossman]). Let $B$ be a finitely generated, conjugacy separable group with Property $A$. Then $\operatorname{Out}(B)$ is $\mathcal{R} \mathcal{F}$.

The next result was first proved in [12, Theorem 3.2] where the proof is quite long. Here we reproduce a slightly modified proof of [13] for the reader's convenience.

Theorem 2.5 ([12, 13]). Let $G=A *_{H} B$, where $A \neq H \neq B$. If $H \subset$ $Z(A) \cap Z(B)$, then $G$ has Property $A$. 
Proof. Let $\bar{G}=G / H=(A / H) *(B / H)$. Then $\bar{G}$ has Property A [2, 9]. Let $\alpha$ be a class-preserving automorphism of $G$. Since $H=Z(G)$, clearly $\alpha(H)=H$. Hence $\bar{\alpha}(\bar{g})=\overline{\alpha(g)}$ is a class-preserving automorphism of $\bar{G}$. Thus $\bar{\alpha}=\operatorname{Inn} \bar{w}$ for some $w \in G$. Therefore, for each $g \in G$, we see that $\alpha(g)=w^{-1} h_{g} g w$ for some $h_{g} \in H$. Let $\alpha^{\prime}=\operatorname{Inn} w^{-1} \circ \alpha$. Then $\alpha^{\prime}$ is a class-preserving automorphism of $G$ and, for each $g \in G, \alpha^{\prime}(g)=h_{g} g$ where $h_{g} \in H$.

Let $b \in B \backslash H$ be fixed and $\alpha^{\prime}(b)=h_{b} b$ for $h_{b} \in H$. For each $x \in A \backslash H$, $x b \sim_{G} \alpha^{\prime}(x) \alpha^{\prime}(b)=h_{x} h_{b} x b$. By Theorem $2.3 x b \sim_{H} h_{x} h_{b} x b$. Since $H \subset Z(G)$, we have $x b=h_{x} h_{b} x b$. Hence $h_{x}=h_{b}^{-1}$ for all $x \in A \backslash H$. Thus, $\alpha^{\prime}(x)=h_{b}^{-1} x$ for all $x \in A \backslash H$. Since $\alpha^{\prime}$ is a class-preserving automorphism of $G$ and $H=Z(G)$, $\alpha^{\prime}(c)=c$ for all $c \in H$.

(1) Suppose $|A / H| \geq 4$ (or $|B / H| \geq 4$ ).

Let $\bar{A}=A / H$ and $a_{1} \in A \backslash H$. Since $|\bar{A}| \geq 4$, there exists $\bar{a}_{2} \in \bar{A}$ such that $1 \neq \bar{a}_{2} \neq \bar{a}_{1}^{ \pm 1}$. Let $a_{3}=a_{1} a_{2}$. Then $\bar{a}_{3} \neq 1$. Thus $a_{i} \notin H$ for all $1 \leq i \leq 3$. By above, $\alpha^{\prime}\left(a_{3}\right)=h_{b}^{-1} a_{3}$ and $\alpha^{\prime}\left(a_{3}\right)=\alpha^{\prime}\left(a_{1}\right) \alpha^{\prime}\left(a_{2}\right)=h_{b}^{-1} a_{1} h_{b}^{-1} a_{2}=h_{b}^{-2} a_{3}$. Hence $h_{b}=1$. Thus $\alpha^{\prime}(x)=x$ for all $x \in A \backslash H$. Since $b \in B \backslash H$ is arbitrary and $h_{b}=1$, we also have $\alpha^{\prime}(b)=b$ for all $b \in B \backslash H$. Hence $\alpha^{\prime}=\operatorname{Inn} 1$.

(2) Suppose $|A / H|<4$ and $|B / H|<4$.

Since $H \subset Z(A), A$ is abelian and $A=\langle a, H\rangle$ for some $a \in A \backslash H$. Similarly, $B$ is abelian and $B=\langle b, H\rangle$ for some $b \in B \backslash H$. Let $\alpha^{\prime}(a)=a h$, where $h \in H$. Clearly $\{a\}^{A} \cap H=\emptyset$. Since $a \sim_{G} \alpha^{\prime}(a)=a h$, by Theorem 2.3 we have $a \sim_{A} a h$. Since $A$ is abelian, $a=a h$. Thus $h=1$, that is $\alpha^{\prime}(a)=a$. Similarly, $\alpha^{\prime}(b)=b$. Hence $\alpha^{\prime}=\operatorname{Inn} 1$.

Hence, $\alpha=\operatorname{Inn} w$. Thus $G$ has Property A.

Throughout the next two sections, we shall use the following hypothesis.

Hypothesis $(*)$ : Suppose $A, B$ are finitely generated nilpotent groups and $p$ is a prime integer. Let $G=A *\langle c\rangle B$, where $\langle c\rangle \triangleleft A, B$ and $|c|=p^{2}$. In addition, we suppose that $A \neq\langle c\rangle \neq B$ throughout the paper.

Remark 2.6. Let $A$ be a nilpotent group. For $1 \neq\langle c\rangle \triangleleft A$, we have $\langle c\rangle \cap Z(A) \neq$ 1. Hence, if $|c|=p^{2}$ ( $p$ is a prime), then $\langle c\rangle \cap Z(A)=\left\langle c^{p}\right\rangle$ or $\langle c\rangle$. Thus $\left\langle c^{p}\right\rangle \subset Z(A)$. Therefore, if $G$ is as in (*), then $\left\langle c^{p}\right\rangle \subset Z(A) \cap Z(B)$.

Lemma 2.7. Let $G$ be as in $(*)$. Let $\alpha$ be a class-preserving automorphism of $G$ such that for each $g \in G, \alpha(g)=g c^{i p}$ for some integer $0 \leq i<p$. Let $X=\{g \in G \mid \alpha(g)=g\}$. Then $X \triangleleft G$ and $|G: X|=1$ or $p$.

Proof. Since $\alpha$ is an automorphism of $G, X$ is a subgroup of $G$. For $g \in G$ and $x \in X, \alpha\left(g^{-1} x g\right)=\alpha(g)^{-1} \alpha(x) \alpha(g)=\left(g c^{i p}\right)^{-1} x g c^{i p}$. By Remark 2.6, $c^{p} \in Z(A) \cap Z(B)$. Hence $\alpha\left(g^{-1} x g\right)=g^{-1} x g$. Thus $X \triangleleft G$.

Let $X_{i}=\left\{g \in G \mid \alpha(g)=g c^{i p}\right\}$, where $0 \leq i<p$. Then $X=X_{0}$ and $G=\cup_{i=0}^{p-1} X_{i}$. Now suppose $X \neq G$. Hence there exists $g \in G$ such that $\alpha(g)=g c^{i p}$ for some $1 \leq i<p$. Let $k$ be a positive integer such that $k i \equiv 1(\bmod p)$. Then $\alpha\left(g^{k}\right)=g^{k} c^{k i p}=g^{k} c^{p}$. Let $a=g^{k}$. Then $\alpha(a)=a c^{p}$ and $\alpha\left(a^{i}\right)=a^{i} c^{i p}$. Hence $a^{i} \in X_{i}$ and $X_{i} \neq \emptyset$ for each $1 \leq i<p$. Let 
$x, y \in X_{i}$. Then $\alpha(x)=x c^{i p}$ and $\alpha(y)=y c^{i p}$. Since $\left\langle c^{p}\right\rangle \leq Z(A) \cap Z(B)$, we have $\alpha\left(x^{-1} y\right)=(\alpha(x))^{-1} \alpha(y)=x^{-1} y$. Hence $x^{-1} y \in X$. Thus $X_{i}$ is a coset of $X$ in $G$. In fact $X_{i}=X a^{i}$ for $1 \leq i<p$. Hence $|G: X|=p$. Therefore, $|G: X|=1$ or $p$.

It is interesting to see that $X_{i}$ defined in the proof of Lemma 2.7 is an $\alpha$-invariant coset of $G$. Let $g \in X_{i}$. Then $\alpha(g)=g c^{i p}$. Since $\alpha$ is a classpreserving automorphism, $\alpha(g)=k_{g}^{-1} g k_{g}$ for some $k_{g} \in G$. Assume $\alpha\left(k_{g}\right)=$ $k_{g} c^{j p}$ for some integer $j$. Then

$$
\alpha(\alpha(g))=\alpha\left(k_{g}\right)^{-1} \alpha(g) \alpha\left(k_{g}\right)=\left(k_{g} c^{j p}\right)^{-1} g c^{i p}\left(k_{g} c^{j p}\right)=k_{g}^{-1} g k_{g} c^{i p}=\alpha(g) c^{i p} .
$$

Hence, $\alpha(g) \in X_{i}$ for each $g \in X_{i}$, which implies that $X_{i}$ is $\alpha$-invariant.

Throughout this paper, $X$ will be used in the meaning of Lemma 2.7.

\section{The case that $|A| \geq 32$ or $|B| \geq 32$}

We begin by studying a special kind of class-preserving automorphism $\alpha$ of $G$ in $(*)$ such that for each $g \in G, \alpha(g)=g c^{i p}$ for some integer $0 \leq i<p$, which plays an important role in this paper.

Lemma 3.1. Let $G$ be as in $(*)$. Let $\alpha$ be a class-preserving automorphism of $G$ such that for each $g \in G, \alpha(g)=g c^{i p}$ for some integer $0 \leq i<p$. Suppose, for each $g \in G, \alpha(g)=k_{g}^{-1} g k_{g}$ for some $k_{g} \in G$.

(1) If there exists $a \in A \backslash\langle c\rangle$ such that $[a, c]=1$ and $\alpha(a)=a$, then we can take $k_{y} \in\langle c\rangle$ for each $y \in B \backslash\langle c\rangle$.

(2) If there exist $a \in A \backslash\langle c\rangle$ and $b \in B \backslash\langle c\rangle$ such that $[a, c]=1=[b, c]$, $\alpha(a)=a$ and $\alpha(b)=b$, then $\alpha(c)=c$ and we can take $k_{x}, k_{y} \in\langle c\rangle$ for each $x \in A$ and $y \in B$.

Proof. (1) Suppose that there exists $a \in A \backslash\langle c\rangle$ such that $[a, c]=1$ and $\alpha(a)=$ $a$. Let $y \in B \backslash\langle c\rangle$. By assumption, $\alpha(y)=y c^{i p}$ for some $0 \leq i<p$. If $i=0$, then we can take $k_{y}=1 \in\langle c\rangle$. Hence we let $1 \leq i<p$. Then $a y \sim_{G} \alpha(a y)=\alpha(a) \alpha(y)=a y c^{i p}$. By Theorem 2.3, ay $\sim_{\langle c\rangle} a y c^{i p}$. Hence $a y c^{i p}=c^{-r} a y c^{r}$ for some $r$. Since $[a, c]=1$, we have $y c^{i p}=c^{-r} y c^{r}$. Since $\alpha(y)=y c^{i p}$, we can take $k_{y}=c^{r} \in\langle c\rangle$.

(2) Let $\alpha(c)=c c^{i p}$ for some $0 \leq i<p$. Let $1+i p=k$. By assumption, there exist $a \in A \backslash\langle c\rangle$ and $b \in B \backslash\langle c\rangle$ such that $[a, c]=1=[b, c], \alpha(a)=a$ and $\alpha(b)=b$. Note that $a b c \sim_{G} \alpha(a b c)=a b c^{k}$. By Theorem 2.3, $a b c \sim_{\langle c\rangle} a b c^{k}$. Since $[a, c]=1=[b, c]$, we have $a b c=a b c^{k}$. Hence $c=c^{k}$. Thus $\alpha(c)=c^{k}=c$. By (1), we can take $k_{y} \in\langle c\rangle$ for all $y \in B$ and $k_{x} \in\langle c\rangle$ for all $x \in A$.

Lemma 3.2. Let $G$ be as in $(*)$. Let $\alpha$ be a class-preserving automorphism of $G$ such that for each $g \in G, \alpha(g)=g c^{i p}$ for some integer $0 \leq i<p$. If $|A| \geq p^{5}$, then there exists $a \in A \backslash\langle c\rangle$ such that $[a, c]=1$ and $\alpha(a)=a$.

Proof. Note that $\left|A: C_{A}(c)\right|$ is the number of conjugate class of $c$ in $A$. Since $\langle c\rangle \triangleleft A,\left|A: C_{A}(c)\right| \leq p^{2}-p$. By Lemma 2.7, $|A: A \cap X|=1$ or $p$. Hence 
$\left|A: X \cap C_{A}(c)\right| \leq p^{3}-p^{2}$. Thus $\left|X \cap C_{A}(c)\right|>p^{2}$. Since $|c|=p^{2}$, there exists $a \in X \cap C_{A}(c) \backslash\langle c\rangle$. It follows that $a \in A \backslash\langle c\rangle,[a, c]=1$ and $\alpha(a)=a$.

From now on, we focus on the case that $|c|=4$.

Lemma 3.3. Let $G$ be as in $(*)$ with $p=2$. Let $\alpha$ be a class-preserving automorphism such that $\alpha(g)=g$ or $g c^{2}$ for each $g \in G$. If $c \notin Z(A)$ and $c \notin Z(B)$, then $\alpha=\operatorname{Inn} 1$ or $\operatorname{Inn} c$.

Proof. Since $c \notin Z(A)$, there exists $a \in A \backslash C_{A}(c)$. Note that $\left|A: C_{A}(c)\right|$ is the number of conjugate class of $c$ in $A$. Since $\langle c\rangle \triangleleft A$ and $|c|=4$, we have $\left|A: C_{A}(c)\right|=2$. Hence, we have $A=\left\langle a, C_{A}(c)\right\rangle$ and $a^{-1} c a=c^{-1}$. Similarly, $\left|B: C_{B}(c)\right|=2$, and there exists $b \in B \backslash C_{B}(c)$, and $B=\left\langle b, C_{B}(c)\right\rangle$ with $b^{-1} c b=c^{-1}$.

We first claim that $\alpha(x) \alpha(y)=x y$ for all $x \in A \backslash C_{A}(c)$ and $y \in B \backslash C_{B}(c)$. Clearly, $\alpha(x) \in A \backslash\langle c\rangle$ and $\alpha(y) \in B \backslash\langle c\rangle$. Since $x y \sim_{G} \alpha(x y)=\alpha(x) \alpha(y)$, by Theorem 2.3, $x y \sim_{\langle c\rangle} \alpha(x) \alpha(y)$. Thus $\alpha(x) \alpha(y)=c^{-r} x y c^{r}$ for some $r$. Since $x \in A \backslash C_{A}(c), x^{-1} c x=c^{-1}$. Similarly, $y^{-1} c y=c^{-1}$ for $y \in B \backslash C_{B}(c)$. Hence $c^{-r} x y c^{r}=x y$. Thus we have $\alpha(x) \alpha(y)=x y$ for all $x \in A \backslash C_{A}(c)$ and $y \in B \backslash C_{B}(c)$.

Now we prove that $\alpha(x)=x$ for all $x \in C_{A}(c)$. Let $x \in C_{A}(c)$. Then $x a \in A \backslash C_{A}(c)$. Hence, by above, $\alpha(x a b)=x a b$ and $\alpha(a b)=a b$. It follows that $\alpha(x)=x$ for all $x \in C_{A}(c)$. Similarly, $\alpha(y)=y$ for all $y \in C_{B}(c)$.

(1) Suppose $\alpha(a)=a$. By above, $A=\left\langle a, C_{A}(c)\right\rangle$ and $\alpha(x)=x$ for all $x \in C_{A}(c)$. Hence, we have $\alpha(x)=x$ for all $x \in A$. Since $\alpha(a) \alpha(b)=a b$, we have $\alpha(b)=b$. Then, as before, $\alpha(y)=y$ for all $y \in B$. Thus $\alpha=\operatorname{Inn} 1$.

(2) Suppose $\alpha(a)=a c^{2}$. By above, $a^{-1} c a=c^{-1}$. Hence $\alpha(a)=a c^{2}=c^{-1} a c$. Since $\alpha(x)=x$ for all $x \in C_{A}(c)$, we have $\alpha(x)=x=c^{-1} x c$ for all $x \in C_{A}(c)$. It follows that $\alpha(x)=c^{-1} x c$ for all $x \in A$. Since $\alpha(a) \alpha(b)=a b$, we have $\alpha(b)=b c^{2}$. Then, as before, $\alpha(y)=c^{-1} y c$ for all $y \in B$. Hence, $\alpha=\operatorname{Inn} c$.

Theorem 3.4. Let $G$ be as in (*) with $p=2$. If $|A|,|B| \geq 2^{5}$, then $G$ has Property A.

Proof. Let $\alpha$ be a class-preserving automorphism of $G$. Consider $\bar{G}=G /\left\langle c^{2}\right\rangle$. Let $\bar{\alpha}$ be the map of $\bar{G}$ such that $\bar{\alpha}(\bar{g})=\overline{\alpha(g)}$. Then $\bar{\alpha}$ is a class-preserving automorphism of $\bar{G}$. Note that $\bar{G}=\bar{A} *\langle\bar{c}\rangle \bar{B}$, where $\bar{A}=A /\left\langle c^{2}\right\rangle$ and $\bar{B}=$ $B /\left\langle c^{2}\right\rangle$. Since $\bar{A}$ is a finitely generated nilpotent group and $\langle\bar{c}\rangle \triangleleft \bar{A}$ with $|\bar{c}|=2$, $\langle\bar{c}\rangle \subset Z(\bar{A})$ (Remark 2.6). Similarly, $\langle\bar{c}\rangle \subset Z(\bar{B})$. Hence, by Theorem 2.5, we see $\bar{\alpha}$ is an inner automorphism of $\bar{G}$. Hence there exists $a \in G$ such that $\bar{\alpha}(\bar{g})=\bar{a}^{-1} \bar{g} \bar{a}$ for all $\bar{g} \in \bar{G}$. Thus, for each $g \in G, \alpha(g)=a^{-1} g c^{2 i} a$ for some $i$. Let $\alpha_{0}=\operatorname{Inn} a^{-1} \circ \alpha$. Then for each $g \in G, \alpha_{0}(g)=g$ or $g c^{2}$. Since we want to prove that $\alpha$ is an inner automorphism, it suffices to prove that $\alpha_{0}$ is an inner automorphism. So we can assume that for each $g \in G, \alpha(g)=g$ or $g c^{2}$. Hence, by Lemma 3.1 and Lemma 3.2, we have $\alpha(c)=c$ and $k_{x}, k_{y} \in\langle c\rangle$ for all $x \in A$ and $y \in B$. 
If $c \in Z(A) \cap Z(B)$, then $G$ has Property A by Theorem 2.5. If $c \notin Z(A)$ and $c \notin Z(B)$, by Lemma 3.3, $\alpha$ is an inner automorphism. Hence, we assume that $c \notin Z(A)$ and $c \in Z(B)$ (The other case $c \in Z(A)$ and $c \notin Z(B)$ is similar). Since $c \notin Z(A)$, as in Lemma 3.3, we have $A=\left\langle a, C_{A}(c)\right\rangle$ and $a^{-1} c a=c^{-1}$.

(1) Suppose $\alpha(a)=a$. Since $k_{x} \in\langle c\rangle$ for all $x \in A$, we have $\alpha(x)=x$ for all $x \in C_{A}(c)$. Since $A=\left\langle a, C_{A}(c)\right\rangle, \alpha(x)=x$ for all $x \in A$. Since $k_{y} \in\langle c\rangle$ for all $y \in B$ and $c \in Z(B), \alpha(y)=y$ for all $y \in B$. Thus $\alpha=\operatorname{Inn} 1$.

(2) Suppose $\alpha(a)=a c^{2}$. Since $a^{-1} c a=c^{-1}$, we have $\alpha(a)=a c^{2}=c^{-1} a c$. Also, $\alpha(x)=x=c^{-1} x c$ for $x \in C_{A}(c)$. Thus $\alpha(x)=c^{-1} x c$ for all $x \in A$. Since $k_{y} \in\langle c\rangle$ for all $y \in B$ and $c \in Z(B), \alpha(y)=y=c^{-1} y c$ for all $y \in B$. Hence $\alpha=\operatorname{Inn} c$.

Lemma 3.5. Let $G$ be as in (*) with $p=2$. Let $\alpha$ be a class-preserving automorphism of $G$ such that $\alpha(g)=g$ or $g c^{2}$ for each $g \in G$. If $|A| \geq 2^{4}$ and $c \in Z(A)$, then $\alpha(x)=x$ for all $x \in A$.

Proof. Let $a_{1} \in A \backslash\langle c\rangle$ and $\bar{A}=A /\langle c\rangle$. Clearly $|\bar{A}| \geq 4$. Hence there exists $\bar{a}_{2} \in \bar{A}$ such that $1 \neq \bar{a}_{2} \neq \bar{a}_{1}^{ \pm 1}$. Let $a_{3}=a_{1} a_{2}$. Then $\bar{a}_{3} \neq 1$ and $\bar{a}_{i} \neq \bar{a}_{j}$ for all $1 \leq i \neq j \leq 3$. Thus $a_{i} \notin\langle c\rangle$ and $a_{i}^{-1} a_{j} \notin\langle c\rangle$ for all $1 \leq i \neq j \leq 3$.

We first claim that $\alpha\left(a_{i}\right)=a_{i}$ for $i=1,2,3$. Suppose $\alpha\left(a_{1}\right)=a_{1}$ and $\alpha\left(a_{2}\right)=a_{2} c^{2}$. Let $b \in B \backslash\langle c\rangle$. Since $\alpha(b)=b$ or $b c^{2}$ and $c^{2} \in Z(G)$, we have $\alpha\left(a_{1} b a_{2} b\right)=\alpha\left(a_{1}\right) \alpha(b) \alpha\left(a_{2}\right) \alpha(b)=c^{2} a_{1} b a_{2} b$. Hence $a_{1} b a_{2} b \sim_{G} \alpha\left(a_{1} b a_{2} b\right)=$ $c^{2} a_{1} b a_{2} b$. By Theorem 2.3, we have $c^{2} a_{1} b a_{2} b \sim\langle c\rangle\left(a_{1} b a_{2} b\right)^{*}$, where $\left(a_{1} b a_{2} b\right)^{*}$ is a cyclic permutation of $a_{1} b a_{2} b$. Hence we have either

(1) $c^{2} a_{1} b a_{2} b=c^{-r}\left(a_{1} b a_{2} b\right) c^{r}$ or

(2) $c^{2} a_{1} b a_{2} b=c^{-r}\left(a_{2} b a_{1} b\right) c^{r}$ for some $r$.

By using $c \in Z(A)$ and $b^{-1} c b=c^{ \pm 1},(1)$ implies $c^{2} a_{1} b a_{2} b=a_{1} b a_{2} b$ and (2) implies $c^{2} a_{1} b a_{2} b=a_{2} b a_{1} b$. Hence we have either $c^{2}=1$ from (1) or $a_{2} \in a_{1}\langle c\rangle$ from (2). Both are impossible. Thus we have either $\alpha\left(a_{1}\right)=a_{1}$ and $\alpha\left(a_{2}\right)=a_{2}$ or $\alpha\left(a_{1}\right)=a_{1} c^{2}$ and $\alpha\left(a_{2}\right)=a_{2} c^{2}$. But if $\alpha\left(a_{1}\right)=a_{1} c^{2}$ and $\alpha\left(a_{2}\right)=a_{2} c^{2}$, then $\alpha\left(a_{3}\right)=\alpha\left(a_{1} a_{2}\right)=a_{1} c^{2} a_{2} c^{2}=a_{1} a_{2}=a_{3}$. Then, by considering $\alpha\left(a_{1}\right)$ and $\alpha\left(a_{3}\right)$, we have a contradiction as before. Therefore, $\alpha\left(a_{i}\right)=a_{i}$ for $i=1,2,3$. Since $a_{1} \in A \backslash\langle c\rangle$ is arbitrary, we have $\alpha(x)=x$ for all $x \in A \backslash\langle c\rangle$.

We shall show that $\alpha(c)=c$. Let $a_{1}, a_{2} \in A$ as above. Since $\alpha(c)=$ $c$ or $c^{3}$, let $\alpha(c)=c^{s}$. Clearly $c a_{1} b a_{2} b \sim_{G} \alpha\left(c a_{1} b a_{2} b\right)=\alpha(c) \alpha\left(a_{1} b a_{2} b\right)=$ $c^{s} a_{1} b a_{2} b$. Hence, we have $c^{s} a_{1} b a_{2} b \sim_{\langle c\rangle}\left(c a_{1} b a_{2} b\right)^{*}$, where $\left(c a_{1} b a_{2} b\right)^{*}$ is a cyclic permutation of $c a_{1} b a_{2} b$. Thus, either (1) $c^{s} a_{1} b a_{2} b=c^{-r}\left(c a_{1} b a_{2} b\right) c^{r}$ or (2) $c^{s} a_{1} b a_{2} b=c^{-r}\left(a_{2} b c a_{1} b\right) c^{r}$. As in above, (2) implies that $a_{1} \in a_{2}\langle c\rangle$, that is, $a_{2}^{-1} a_{1} \in\langle c\rangle$, which is impossible. Hence we have $c^{s}=c$ from (1). Thus $\alpha(c)=c^{s}=c$. Therefore, $\alpha(x)=x$ for all $x \in A$.

Theorem 3.6. Let $G$ be as in $(*)$ with $p=2$. If $|A| \geq 2^{5}$ and $|B|=4 p_{1} p_{2}$ for primes $p_{1}, p_{2}$, then $G$ has Property $A$. 
Proof. Let $\alpha$ be a class-preserving automorphism of $G$. As in Theorem 3.4, we assume that $\alpha(g)=g$ or $g c^{2}$ for each $g \in G$. By Theorem 2.5 and Lemma 3.3, we consider the following two cases.

Case 1. $c \notin Z(A)$ and $c \in Z(B)$.

As in Lemma 3.3, we have $A=\left\langle a, C_{A}(c)\right\rangle$ where $a^{-1} c a=c^{-1}$. By Lemma 3.5, $\alpha(y)=y$ for all $y \in B$. Let $x \in C_{A}(c) \backslash\langle c\rangle$. Since $x b \sim_{G} \alpha(x b)$ and $[x, c]=$ $1=[b, c]$, as before, we have $\alpha(x) \alpha(b)=x b$. Since $\alpha(b)=b, \alpha(x)=x$ for $x \in C_{A}(c) \backslash\langle c\rangle$.

Now if $\alpha(a)=a$, then $\alpha=\operatorname{Inn} 1$. If $\alpha(a)=a c^{2}$, then $\alpha(a)=c^{-1} a c$. Since $\alpha(x)=x=c^{-1} x c$ for $x \in C_{A}(c)$ and $\alpha(y)=y=c^{-1} y c$ for all $y \in B$, we have $\alpha=\operatorname{Inn} c$.

Case 2. $c \in Z(A)$ and $c \notin Z(B)$.

As in Lemma 3.3, we have $B=\left\langle b, C_{B}(c)\right\rangle$, where $\left|B: C_{B}(c)\right|=2$ and $b^{-1} c b=c^{-1}$. Let $b_{1} \in C_{B}(c) \backslash\langle c\rangle$. Since $\left|C_{B}(c)\right|=2 p_{1} p_{2}$ and $|c|=4, C_{B}(c)=$ $\left\langle b_{1}, c\right\rangle$. By Lemma 3.5, $\alpha(x)=x$ for all $x \in A$ and, as in Case 1 above, $\alpha\left(b_{1}\right)=b_{1}$. Thus, if $\alpha(b)=b$, then $\alpha=\operatorname{Inn} 1$. If $\alpha(b)=b c^{2}$, then $\alpha(b)=c^{-1} b c$. Since $B=\left\langle b, C_{B}(c)\right\rangle$ and $\alpha\left(b_{1}\right)=b_{1}=c^{-1} b_{1} c$, we have $\alpha(y)=c^{-1} y c$ for all $y \in B$. Clearly $\alpha(x)=x=c^{-1} x c$ for all $x \in A$. Hence $\alpha=\operatorname{Inn} c$.

Theorem 3.7. Let $G$ be as in $(*)$ with $p=2$. If $|A| \geq 2^{5}$ and $|B|=4 p_{1}$ for some prime $p_{1}$, then $G$ has Property $A$.

Proof. Let $\alpha$ be a class-preserving automorphism of $G$. As before, we assume that $\alpha(g)=g$ or $g c^{2}$ for each $g \in G$. By Theorem 2.5 and Lemma 3.3, we consider the following two cases.

Case 1. $c \notin Z(A)$ and $c \in Z(B)$.

As before, let $A=\left\langle a, C_{A}(c)\right\rangle$ where $a^{-1} c a=c^{-1}$. Let $b \in B \backslash\langle c\rangle$. Then $B=\langle b, c\rangle$ is abelian. Let $y \in B \backslash\langle c\rangle$. Clearly $y \sim_{G} \alpha(y)$. Since $B$ is abelian, $\{y\}^{B} \cap\langle c\rangle=\emptyset$. Hence, by Theorem 2.3,y $\sim_{B} \alpha(y)$. Thus $\alpha(y)=y$ for all $y \in B \backslash\langle c\rangle$. In particular, $\alpha(b)=b$ and $\alpha(b c)=b c$. Hence, $\alpha(c)=c$. Let $x \in C_{A}(c) \backslash\langle c\rangle$. Clearly $x b \sim_{G} \alpha(x b)=\alpha(x) b$. By Theorem 2.3, $x b \sim_{\langle c\rangle} \alpha(x) b$. Since $[x, c]=1=[b, c]$, as before, we have $\alpha(x) b=x b$. Hence $\alpha(x)=x$ for $x \in C_{A}(c) \backslash\langle c\rangle$.

Now if $\alpha(a)=a$, then $\alpha=\operatorname{Inn} 1$. If $\alpha(a)=a c^{2}$, then $\alpha(a)=c^{-1} a c$. Hence, as before, $\alpha=\operatorname{Inn} c$.

Case 2. $c \in Z(A)$ and $c \notin Z(B)$.

As in Case 1 , let $B=\langle b, c\rangle$, where $b^{-1} c b=c^{-1}$. By Lemma 3.5, we have $\alpha(x)=x$ for all $x \in A$. If $\alpha(b)=b$, then $\alpha=\operatorname{Inn} 1$. If $\alpha(b)=b c^{2}$, then $\alpha(b)=c^{-1} b c$. Hence, as before, $\alpha=\operatorname{Inn} c$.

\section{The case that $|A|,|B|<32$}

In this section, we show that $G=A *\langle c\rangle$, where $\langle c\rangle \triangleleft A, B$ and $|c|=4$, has property $\mathrm{A}$, if $|A|,|B|<32$. In fact, we prove this in several cases in a little bit generalized form. 
Theorem 4.1. Let $G$ be as in $(*)$ with $p=2$. If $|A|=4 p_{1}$ and $|B|=4 p_{2}$ for primes $p_{1}, p_{2}$, then $G$ has Property $A$.

Proof. Let $\alpha$ be a class-preserving automorphism of $G$. As before, we may assume that $\alpha(g)=g$ or $g c^{2}$ for each $g \in G$. If $c \in Z(A) \cap Z(B)$, then $G$ has Property A by Theorem 2.5. If $c \notin Z(A)$ and $c \notin Z(B)$, then by Lemma 3.3, $\alpha$ is an inner automorphism. Hence we need only consider the case that $c \in Z(A)$ and $c \notin Z(B)$ (The other case that $c \notin Z(A)$ and $c \in Z(B)$ is similar).

Let $a \in A \backslash\langle c\rangle$. Since $|A|=4 p_{1}$ and $|c|=4$, we have $A=\langle a, c\rangle$. Since $c \in Z(A), A$ is abelian. As in the proof of Lemma 3.3, $B=\langle b, c\rangle$, where $b \notin\langle c\rangle$ and $b^{-1} c b=c^{-1}$.

We claim that $\alpha(x)=x$ for $x \in A \backslash\langle c\rangle$. Since $A$ is abelian and $x \in A \backslash\langle c\rangle$, we have $\{x\}^{A} \cap\langle c\rangle=\emptyset$. Since $x \sim_{G} \alpha(x)$ and $\alpha(x)$ is cyclically reduced, by Theorem 2.3, $x \sim_{A} \alpha(x)$. Since $A$ is abelian, we have $\alpha(x)=x$ for $x \in A \backslash\langle c\rangle$. In particular, $\alpha(a)=a$ and $\alpha(a c)=a c$. Since $\alpha(a c)=\alpha(a) \alpha(c)=a \alpha(c)$, we have $\alpha(c)=c$.

Now we have either $\alpha(b)=b$ or $\alpha(b)=b c^{2}$. If $\alpha(b)=b$, then $\alpha=\operatorname{Inn} 1$. If $\alpha(b)=b c^{2}$, then $\alpha(b)=c^{-1} b c$. Hence $\alpha(y)=c^{-1} y c$ for all $y \in B$. Since $c \in Z(A)$, we have $\alpha(x)=x=c^{-1} x c$ for all $x \in A$. Hence, $\alpha=\operatorname{Inn} c$.

Theorem 4.2. Let $G$ be as in $(*)$ with $p=2$. If $|A|=4 p_{1} p_{2}$ and $|B|=4 p_{3}$ for primes $p_{1}, p_{2}, p_{3}$, then $G$ has Property $A$.

Proof. Let $\alpha$ be a class-preserving automorphism of $G$. As before, we assume that $\alpha(g)=g$ or $g c^{2}$ for each $g \in G$. If $c \in Z(A) \cap Z(B)$, then $G$ has Property A by Theorem 2.5. If $c \notin Z(A)$ and $c \notin Z(B)$, by Lemma 3.3, $\alpha$ is an inner automorphism. Hence, we consider the following cases.

Case 1. $c \notin Z(A)$ and $c \in Z(B)$.

Since $c \notin Z(A)$, there exists $a \in A \backslash C_{A}(c)$. As in the proof of Lemma 3.3, $\left|A: C_{A}(c)\right|=2$ and $A=\left\langle a, C_{A}(c)\right\rangle$, where $a^{-1} c a=c^{-1}$. Hence $\left|C_{A}(c)\right|=$ $2 p_{1} p_{2}$. Since $|c|=4$, there exists $a_{1} \in C_{A}(c) \backslash\langle c\rangle$. Then $C_{A}(c)=\left\langle a_{1}, c\right\rangle$. Hence $A=\left\langle a, a_{1}, c\right\rangle$, where $a^{-1} c a=c^{-1}$ and $\left[a_{1}, c\right]=1$.

Let $b \in B \backslash\langle c\rangle$. Then $B=\langle b, c\rangle$ and $B$ is abelian $(c \in Z(B))$. As in the proof of Theorem 4.1, $\alpha(y)=y$ for all $y \in B \backslash\langle c\rangle$. In particular, $\alpha(b)=b$ and $\alpha(b c)=b c$. Hence $\alpha(c)=c$. Thus $\alpha(y)=y$ for all $y \in B$.

Clearly $a_{1} b \sim_{G} \alpha\left(a_{1}\right) \alpha(b)=\alpha\left(a_{1}\right) b$. By Theorem 2.3, $a_{1} b \sim_{\langle c\rangle} \alpha\left(a_{1}\right) b$. Since $\left[a_{1}, c\right]=[b, c]=1$, we have $a_{1} b=\alpha\left(a_{1}\right) b$. Hence $\alpha\left(a_{1}\right)=a_{1}$.

Hence, if $\alpha(a)=a$, then $\alpha=\operatorname{Inn} 1$. If $\alpha(a)=a c^{2}$, then $\alpha(a)=a^{-1} c a$. Clearly $\alpha\left(a_{1}\right)=a_{1}=c^{-1} a_{1} c$ and $\alpha(y)=y=c^{-1} y c$ for all $y \in B$. Hence $\alpha=\operatorname{Inn} c$.

Case 2. $c \in Z(A)$ and $c \notin Z(B)$.

By Lemma 3.5, $\alpha(x)=x$ for all $x \in A$. As before, we have $B=\langle b, c\rangle$, where $b^{-1} c b=c^{-1}$. If $\alpha(b)=b$, then clearly $\alpha=\operatorname{Inn} 1$. If $\alpha(b)=b c^{2}$, then $\alpha(b)=c^{-1} b c$. Since $\alpha(x)=x=c^{-1} x c$ for all $x \in A$, we have $\alpha=\operatorname{Inn} c$. 
Theorem 4.3. Let $G$ be as in $(*)$ with $p=2$. If $|A|=4 p_{1} p_{2}$ and $|B|=4 p_{3} p_{4}$ for primes $p_{1}, p_{2}, p_{3}, p_{4}$, then $G$ has Property $A$.

Proof. Let $\alpha$ be a class-preserving automorphism of $G$. As before, we assume that $\alpha(g)=g$ or $g c^{2}$ for each $g \in G$. If $c \in Z(A) \cap Z(B)$, then $G$ has Property A by Theorem 2.5. If $c \notin Z(A)$ and $c \notin Z(B)$, by Lemma 3.3, $\alpha$ is an inner automorphism. Hence we assume $c \notin Z(A)$ and $c \in Z(B)$ (The case that $c \in Z(A)$ and $c \notin Z(B)$ is similar).

As in Case 1 in Theorem 4.2, we have $A=\left\langle a, a_{1}, c\right\rangle$, where $a^{-1} c a=c^{-1}$ and $\left[a_{1}, c\right]=1$. Since $c \in Z(B)$ and $|B| \geq 16$, by Lemma $3.5, \alpha(y)=y$ for all $y \in B$. As in Case 1 of Theorem 4.2, we can show that $\alpha\left(a_{1}\right)=a_{1}$. Thus, if $\alpha(a)=a$, then $\alpha=\operatorname{Inn} 1$. If $\alpha(a)=a c^{2}$, then $\alpha(a)=c^{-1} a c$. Hence, as before, $\alpha=\operatorname{Inn} c$.

\section{Conclusion}

Theorem 5.1. Let $A, B$ be finitely generated nilpotent groups. Let $G=A *\langle c\rangle$ $B$, where $\langle c\rangle \triangleleft A, B$ and $A \neq\langle c\rangle \neq B$. If $|c|=2 p$ for an odd prime $p$, then $G$ has Property $A$.

Proof. Clearly $\left|c^{p}\right|=2$. Since $c^{p}$ is the only element of order 2 in $\langle c\rangle, a^{-1} c^{p} a=$ $c^{p}$ for all $a \in A$. Hence $c^{p} \in Z(A)$. Similarly, $c^{p} \in Z(B)$. By considering $\bar{G}=G /\left\langle c^{p}\right\rangle=\bar{A} *\langle\bar{c}\rangle \bar{B}$, where $\bar{A}=A /\left\langle c^{p}\right\rangle$ and $\bar{B}=B /\left\langle c^{p}\right\rangle$, as in the proof of Theorem 3.4, we shall show that every class-preserving automorphism $\alpha$ of $G$ such that, for each $g \in G, \alpha(g)=g$ or $g c^{p}$, is inner.

Let $\alpha$ be a class-preserving automorphism of $G$ such that, for each $g \in G$, $\alpha(g)=g$ or $g c^{p}$. We first claim that $\alpha(x) \alpha(y)=x y$ for all $x \in A \backslash\langle c\rangle$ and $y \in B \backslash\langle c\rangle$. Since $\langle c\rangle \triangleleft A$, let $x^{-1} c x=c^{\epsilon}$, where $(\epsilon, 2 p)=1$ and $1 \leq \epsilon<2 p$. Similarly, let $y^{-1} c y=c^{\delta}$, where $(\delta, 2 p)=1$ and $1 \leq \delta<2 p$. Hence, for each integer $r$, we have $c^{-r} x y c^{r}=x c^{-r \epsilon} y c^{r}=x y c^{-r \epsilon \delta} c^{r}=x y c^{(1-\epsilon \delta) r}$. Now $c^{(1-\epsilon \delta) r} \in\left\langle c^{2}\right\rangle$ (because $\epsilon, \delta$ are odd). Since $x y \sim_{G} \alpha(x y)=\alpha(x) \alpha(y)$, by Theorem 2.3 we have $x y \sim\langle c\rangle \alpha(x) \alpha(y)$. Thus $\alpha(x) \alpha(y)=c^{-r} x y c^{r}$ for some $r$. By above, we have $\alpha(x) \alpha(y)=x y c^{(1-\epsilon \delta) r}$. Clearly $c^{p} \notin\left\langle c^{2}\right\rangle$. Since $\alpha(x) \alpha(y)=$ $\alpha(x y)=x y$ or $x y c^{p}$, we must have $\alpha(x y)=x y$.

By above, $\alpha(x y c)=x y c$ for $x \in A \backslash\langle c\rangle$ and $y \in B \backslash\langle c\rangle$. Since $\alpha(x y)=x y$, $\alpha(c)=c$.

Case 1. $|A| \geq 6 p$ (or, similarly, $|B| \geq 6 p$ ).

Let $a \in A \backslash\langle c\rangle$ be arbitrary. Let $\bar{A}=A /\langle c\rangle$. Then $|\bar{A}| \geq 3$. Hence there exists $\bar{u} \in \bar{A}$ such that $1 \neq \bar{u} \neq \bar{a}^{-1}$. Then $u \notin\langle c\rangle$ and $a u \notin\langle c\rangle$. By above, $\alpha($ auy $)=a u y$ and $\alpha(u y)=u y$ for $y \in B \backslash\langle c\rangle$. Hence $\alpha(a)=a$. This proves that $\alpha(x)=x$ for all $x \in A \backslash\langle c\rangle$. Since $\alpha(x) \alpha(y)=x y$ by above, $\alpha(y)=y$ for all $y \in B \backslash\langle c\rangle$. Therefore $\alpha=\operatorname{Inn} 1$.

Case 2. $|A|=4 p$ and $|B|=4 p$. Since $\langle c\rangle \triangleleft A$ and $|c|=2 p$, there exists $a \in A \backslash\langle c\rangle$ such that $A=\langle a, c\rangle$. Similarly, there exists $b \in B \backslash\langle c\rangle$ such that $B=\langle b, c\rangle$. 
Subcase 1. $c \notin Z(A)$ and $c \in Z(B)$ (similarly, $c \in Z(A)$ and $c \notin Z(B)$ ). Clearly $B$ is abelian. Since $\alpha(b) \sim_{G} b$, by Theorem 2.3 we have $\alpha(b) \sim_{B} b$. Hence $\alpha(b)=b$. Since $\alpha(a b)=a b, \alpha(a)=a$. Hence $\alpha=\operatorname{Inn} 1$.

Subcase 2. $c \notin Z(A)$ and $c \notin Z(B)$. Suppose $\alpha(a)=a c^{p}$. Let $\alpha(a)=$ $k_{a}^{-1} a k_{a}$ for $k_{a} \in G$. Let $k_{a}=u_{1} \cdots u_{s}$ be an alternating product in $G=A *_{\langle c\rangle} B$. Then $a c^{p}=u_{s}^{-1} \cdots u_{1}^{-1} a u_{1} \cdots u_{s}$. Since $a \notin\langle c\rangle, u_{1}^{-1} a u_{1} \notin\langle c\rangle$. Hence we have $u_{1} \in A$ and $r=1$. Let $u_{1}=a^{i} c^{j}$ for some $i, j$. Then $\alpha(a)=u_{1}^{-1} a u_{1}=c^{-j} a c^{j}$. Let $a^{-1} c a=c^{\lambda}$, where $(\lambda, 2 p)=1$ and $1 \leq \lambda<2 p$. Then $\alpha(a)=c^{-j} a c^{j}=$ $a c^{(1-\lambda) j}$. Since $\lambda$ is odd, $c^{(1-\lambda) j} \in\left\langle c^{2}\right\rangle$. Hence $\alpha(a)=c^{-j} a c^{j}=a c^{(1-\lambda) j} \in$ $a\left\langle c^{2}\right\rangle$. This contradicts our assumption $\alpha(a)=a c^{p}$. Hence $\alpha(a)=a$. Thus $\alpha(b)=b$, since $\alpha(a b)=a b$. Therefore, $\alpha=\operatorname{Inn} 1$.

Corollary 5.2. Let $A, B$ be finitely generated nilpotent groups. Let $G=A *\langle c\rangle$ $B$, where $\langle c\rangle \triangleleft A, B$ and $A \neq\langle c\rangle \neq B$. If $|c|<8$, then $G$ has Property $A$.

Proof. If $|c|=p$ for a prime integer $p$, then $\langle c\rangle \cap Z(A) \neq 1$. Hence $c \in Z(A)$. Similarly, $c \in Z(B)$. Thus, by Theorem 2.5, $G$ has Property A if $|c|=p$ for a prime $p$. For $|c|=4$, by theorems in Sections 3 and $4, G$ has Property A. Theorem 5.1 shows that $G$ has Property A if $|c|=6$. Hence $G$ has Property A if $|c|<8$.

Since the generalized free products of finitely generated nilpotent groups, amalgamating a cyclic subgroup, are conjugacy separable [4], combining with Corollary 5.2 and Theorem 2.4, we have the following.

Theorem 5.3. Let $A, B$ be finitely generated nilpotent groups. Let $G=A *\langle c\rangle$ $B$, where $\langle c\rangle \triangleleft A, B$ and $A \neq\langle c\rangle \neq B$. If $|c|<8$, then Out $(G)$ is residually finite.

It is interesting to see that the result is not true even when the amalgamating normal subgroup is of order 8 by the following example [13].

Example 5.4. Consider the following groups isomorphic to $C_{8} \rtimes \operatorname{Aut}\left(C_{8}\right)$.

$$
\begin{aligned}
& A=\left\langle x, y, z: x^{8}=y^{2}=z^{2}=[y, z]=1, x^{y}=x^{-1}, x^{z}=x^{5}\right\rangle, \\
& B=\left\langle x, y_{1}, z_{1}: x^{8}=y_{1}^{2}=z_{1}^{2}=\left[y_{1}, z_{1}\right]=1, x^{y_{1}}=x^{-1}, x^{z_{1}}=x^{5}\right\rangle .
\end{aligned}
$$

The map $\phi: x \rightarrow x, y \rightarrow y, z \rightarrow x^{4} z$ defines a class-preserving automorphism of $A$ which is not inner [7]. Similarly, the map $\phi_{1}: x \rightarrow x, y_{1} \rightarrow y_{1}, z_{1} \rightarrow x^{4} z_{1}$ defines a class-preserving automorphism of $B$.

Let $G=A *\langle x\rangle B$. Then the map $\varphi: x \rightarrow x, y \rightarrow y, z \rightarrow x^{4} z, y_{1} \rightarrow$ $y_{1}, z_{1} \rightarrow x^{4} z_{1}$ defines an automorphism of $G$. It was proved in [13] that $\varphi$ is a class-preserving automorphism of $G$ which is not inner.

Using the above example, it is not difficult to construct generalized free products amalgamating a normal subgroup of order greater than 8 which have not Property A. 
Example 5.5. Let $A, B$ and $G$ be as above. Consider

$$
G_{1}=(A \times\langle d\rangle) *_{H}(B \times\langle d\rangle),
$$

where $H=\langle x\rangle \times\langle d\rangle$ and $|d|=n$. We note that if $n$ is odd, then $H$ is cyclic. Since $\langle x\rangle$ is normal in both $A$ and $B, H=\langle x\rangle \times\langle d\rangle$ is normal in $A \times\langle d\rangle$ and in $B \times\langle d\rangle$. Then it is clear that $G_{1}=G \times\langle d\rangle$. Since $G$ has not Property A, $G_{1}$ has not Property A.

\section{References}

[1] R. B. J. T. Allenby, G. Kim, and C. Y. Tang, Residual finiteness of outer automorphism groups of certain pinched 1-relator groups, J. Algebra 246 (2001), no. 2, 849-858.

[2] _ _ Residual finiteness of outer automorphism groups of finitely generated nontriangle Fuchsian groups, Internat. J. Algebra Comput. 15 (2005), no. 1, 59-72.

[3] W. Burnside, On the outer automorphisms of a group, Proc. London Math. Soc. 11 (1913), 40-42.

[4] J. L. Dyer, Separating conjugates in amalgamated free products and HNN extensions, J. Austral. Math. Soc. Ser. A 29 (1980), no. 1, 35-51.

[5] G. Endimioni, Pointwise inner automorphisms in a free nilpotent group, Q. J. Math. 53 (2002), no. 4, 397-402.

[6] E. K. Grossman, On the residual finiteness of certain mapping class groups, J. London Math. Soc. (2) 9 (1974), 160-164.

[7] S. Jackowski and Z. Marciniak, Group automorphisms inducing the identity map on cohomology, J. Pure Appl. Algebra 44 (1987), no. 1-3, 241-250.

[8] W. Magnus, A. Karrass, and D. Solitar, Combinatorial Group Theory, Pure and Applied Math. Vol. XIII, Wiley-Interscience, New York-London-Sydney, 1966.

[9] M. V. Neshadim, Free products of groups that do not have outer normal automorphisms, Algebra and Logic 35 (1996), no. 5, 316-318.

[10] D. Segal, On the outer automorphism group of a polycyclic group, In Proc. of the Second International Group Theory Conference (Bressanone, 1989), Rend. Circ. Mat. Palermo (2) Suppl. No. 23 (1990), 265-278.

[11] G. E. Wall, Finite groups with class-preserving outer automorphisms, J. London Math. Soc. 22 (1947), 315-320.

[12] P. C. Wong and K. B. Wong, Residual finiteness of outer automorphism groups of certain tree products, J. Group Theory 10 (2007), no. 3, 389-400.

[13] W. Zhou and G. Kim, Class-preserving automorphisms and inner automorphisms of certain tree products of groups, J. Algebra 341 (2011), 198-208.

Wei ZHOU

School of Mathematics and Statistics

SOUTHWEST UNIVERSITY

Chongqing 400715, P. R. China

E-mail address: zh_great@swu.edu.cn

GOANSU Kim

Department of Mathematics

YeUNGNAM UNIVERSITY

KYONGSAN 712-749, KOREA

E-mail address: gskim@yu.ac.kr 